\title{
ASPECTOS PRÁTICOS RELACIONADOS AO USO DO RINCOFOROL, O FEROMÔNIO DE AGREGAÇÃO DA BROCA-DO-OLHO-DO-COQUEIRO RHYNCHOPHORUS PALMARUM L. (COLEOPTERA: CURCULIONIDAE) NO CONTROLE DE PRAGAS DO COQUEIRO. ANÁlISE DE SUA EFICIÊNCIA EM CAMPO.
}

\author{
Daniela Maria do Amaral Ferraz Navarro", Maria Márcia Murta ${ }^{\&}$, Adriana Guimarães Duarte, Ivanildo Soares de Lima, \\ Ruth Rufino do Nascimento e Antônio Euzébio Goulart Sant'Ana* \\ Departamento de Química/CCEN - Universidade Federal de Alagoas, 57072-970, Maceió - AL
}

Recebido em 3/10/00; aceito em 6/7/01

\begin{abstract}
PRACTICAL ASPECTS CONCERNING THE USE OF RHYNCOPHOROL, THE AGGREGATION PHEROMONE OF Rhynchophorus palmarum L. (Coleoptera: Curculionidae) IN THE CONTROL OF COCONUT PESTS. ANALYSIS OF ITS FIELD PERFORMANCE. Rhynchophorol (6-Methyl-2-hepten-4-ol) is the major constituent of the aggregation pheromone of Rhynchophorus palmarum L. (Coleoptera: Curculionidae), which is released by males of this species. The racemic mixture has been produced in one step-simplified preparation. The pheromone is placed into ampoules and furnished to coconut farmers. In the field, traps baited with Rhynchophorol and pieces of sugarcane attract both males and females, reducing the population of $R$. palmarum. The best conditions for field application are showed.
\end{abstract}

Keywords: rhynchophorol; Rhynchophorus palmarum; aggregation pheromone.

\section{INTRODUÇÃO}

O estudo de semioquímicos (sinais químicos) envolvidos nas relações planta-inseto e inseto-inseto tem despertado um grande interesse na comunidade científica nos últimos anos ${ }^{1}$. Isto tem ocorrido devido à crescente preocupação de organizações nacionais e internacionais com o impacto ambiental causado por resíduos de pesticidas e agrotóxicos empregados no controle de pragas, utilizados já há várias décadas e em grande escala, para o aumento da produtividade agrícola. O uso de semioquímicos, como forma alternativa no controle de pragas, proporciona uma série de vantagens, entre as quais: simplicidade, economia e um menor impacto ambiental ${ }^{2}$. Dentre os semioquímicos, podemos citar os feromônios, que são substâncias voláteis envolvidas nas interações intra-específicas dos insetos. Dos feromônios mais investigados nas relações entre insetos, podemos citar $^{3}$ :

Feromônio Sexual: aumenta a probabilidade de sucesso no acasalamento. Dependendo da espécie tanto o macho quanto a fêmea podem produzi-lo.

Feromônio de Trilha: são comuns nos insetos sociais e utilizados para retornar à colônia, empregado para indicar a fonte de alimento ou o novo sítio de moradia onde a colônia deverá se estabelecer.

Feromônio de Território ou Marcação: delimita territórios, minimizando ou até evitando encontros indesejáveis e agressivos.

Feromônio de Alarme: transmite uma mensagem de perigo ou assinala a presença de um inimigo.

Feromônio de Oviposição: auxilia as fêmeas a localizarem um local adequado para postura de ovos.

Feromônio de Agregação: tem como função principal atrair um elevado número de indivíduos da mesma espécie, geralmente com o objetivo de indicar a presença de alimento.

\footnotetext{
* e-mail: aegs@qui.ufal.br

Endereços atuais: "Departamento de Química Fundamental/CCEN Universidade Federal de Pernambuco, 50670-901 Recife-PE. \&Departamento de Química - Universidade de Brasília, 70910-900 Brasília-DF
}

Um exemplo de sucesso no emprego de semioquímico sintético para o controle de pragas no Brasil é o uso do Rincoforol (6-Metil2-hepten-4-ol), feromônio de agregação da Broca-do-olho do coqueiro, Rhynchophorus palmarum L. (Coleoptera: Curculionidae). Outros feromônios de agregação de pragas relacionadas a palmáceas, com importância comercial e/ou ornamental, são descritos e comercializados em outros países para o controle das respectivas $\operatorname{pragas}^{4-7}$ (Tabela 1).

Este artigo tem como objetivo mostrar a importância e eficiência da utilização, em campo, do feromônio de agregação, ( \pm )-Rincoforol, no controle da praga do coqueiro, Rhynchophorus palmarum. Paralelamente, faz-se uma breve descrição do processo de sua identificação estrutural e síntese.

\section{IMPORTÂNCIA, DESCRIÇÃO E BIOLOGIA DO Rhynchophorus palmarum}

O R. palmarum é uma praga importante das plantações de coco (Cocus nucífera) na Venezuela, México, Brasil e áreas do Caribe ${ }^{8}$. Esta cultura é uma das mais importantes no Norte e Nordeste brasileiro ${ }^{9}$, e responde por aproximadamente $80 \%$ da produção nacional. Devemos também citar que o $R$. palmarum pode ainda utilizar como planta hospedeira, ou fonte de alimento, a cana-de-açúcar (Saccharum officinarum), o dendezeiro (Elaeis guineensis), o mamoeiro (Carica papaya), o abacaxizeiro (Ananas comosus), dentre outros ${ }^{10}$, levando a perdas e danos consideráveis às respectivas plantações.

$\mathrm{O}$ inseto adulto (Figura 1) é um besouro de cor negra, cabeça pequena e alongada para a frente em forma de rostro, de hábito diurno e crepuscular. As larvas são de coloração branca e se alimentam do meristema apical da planta, o que permite a instalação de bactérias e outros microorganismos no interior da planta, prejudicando assim, o seu desenvolvimento. R. palmarum é um inseto que apresenta metamorfose completa, ou seja, o ovo origina a larva que passa para a fase de pupa e posteriormente atinge a fase adulta. No estágio adulto, o macho, quando na planta hospedeira, libera o feromônio de agregação que atrai tanto machos como fêmeas, geralmente para a fonte de alimento. Uma vez que ambos os sexos encontram-se no 
Tabela 1. Feromônios de Agregação de diferentes espécies de Rhynchophorus ${ }^{4-6,23}$ apresentados estruturalmente em sua forma racêmica.

Feromônio de Agregação
R. palmarum<smiles>CCCC(C)C(O)CCC</smiles><smiles>CCCCC(C)C(O)CCC</smiles>

Palmeira Asiática

4-Metilnonan-5-ol (Ferruginol)<smiles>CCCCC(=O)C(C)CCC</smiles>

Palmeira Asiática

R. vulneratus

R. ferrugineus

4-Metil-5-nonano

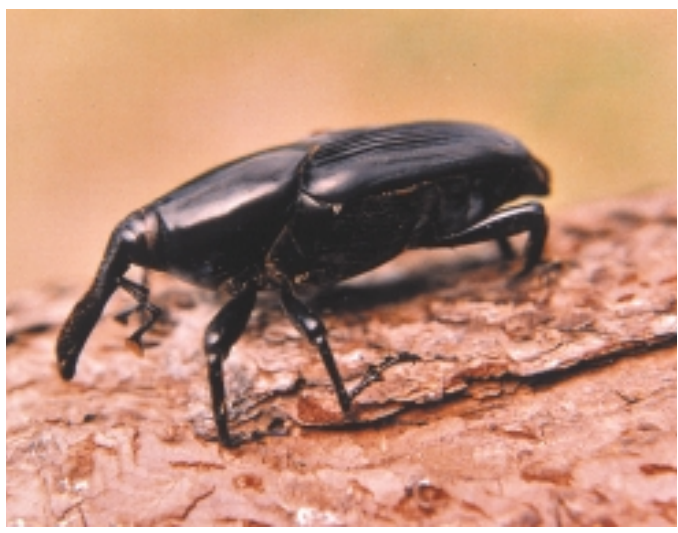

Figura 1. Macho do R. palmarum, responsável pela liberação de feromônios de agregação.

mesmo ambiente, ocorre também a cópula. As fêmeas fazem posturas em ferimentos recém abertos na planta, reiniciando todo o ciclo de vida do $R$. palmarum. Este inseto é o transmissor do nematóide Bursaphelenchus cocophilus (Cobb) ${ }^{11}$, agente causador da doença conhecida como anel vermelho (Figura $2 \mathrm{~A}$ ). Esta doença é letal para o coqueiro e outras palmeiras. A doença no coqueiro pode ser detectada pelo amarelecimento das folhas mais novas (Figura 2 B). Em estágios mais avançados, ocorre apodrecimento do meristema apical e secagem da flecha, com a conseqüente morte da planta ${ }^{12}$.

\section{Rincoforol}

O Rincoforol foi isolado e identificado em 1981 por Rochat e colaboradores $^{13}$, empregando-se, inicialmente, neste processo, entre outros, as técnicas de aeração e eletroantenografia. Entretanto, para a sua completa identificação estrutural, foram, posteriormente empregadas, a cromatografia gasosa e a cromatografia gasosa acoplada à espectrometria de massas ${ }^{14}$.

A estereoquímica absoluta do Rincoforol natural ${ }^{14-15}$ foi definida como sendo o $S(-)$ (2E)-6-metil-2-hepten-4-ol, através da análise comparativa por cromatografia gasosa em coluna capilar (DB-1) do derivado do éster $(S)$-O-acetil-lactato do Rincoforol sintético com éster (S)-O-acetil-lactato produzido a partir do Rincoforol obtido do inseto macho.

A última síntese efetuada para o enantiômero ativo foi desenvolvida pelo Prof. K. Mori em sete etapas, com um rendimento global de $6,6 \%$ e está representada no Esquema $1{ }^{16}$. Partindo do ( \pm )-6-metil-2heptin-4-ol (1), preparado a partir do 3-metilbutanal e propino, uma acetilação com subsequiente hidrólise assimétrica com lipase A (Amano) resultou no $(S)$-1 com 78\% de excesso enantiomérico (e.e.). A sequiência de acetilação e hidrólise foi repetida para melhorar ainda mais o excesso enantiomérico, que passou a ser de $94 \%$ para $(S)$-1. Na tentativa de purificar o enantiômero $(S)-1(65 \%)$ por cromatografia e recristalização, foi preparado o éster 3,5-dinitrobenzoato, $(S)$-3 (92 $\%)$. A etapa de desproteção com $\mathrm{K}_{2} \mathrm{CO}_{3} / \mathrm{MeOH} / \mathrm{CH}_{2} \mathrm{Cl}_{2}$, regenerou (S)-1 com $68 \%$ e $98 \%$ e.e., estimado por HPLC. A última etapa, de hidrogenação, resultou no $(S)$-Rincoforol, $61 \%$ e $98 \%$ e.e.. 
(a)

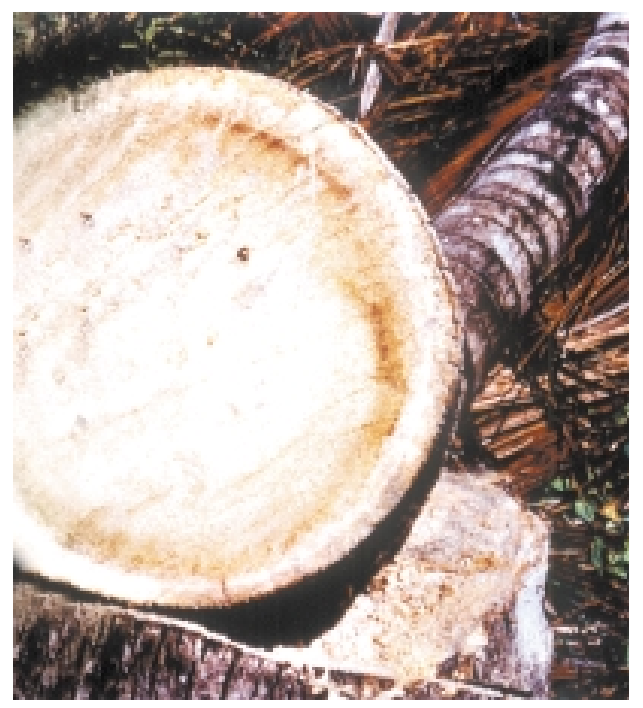

(b)

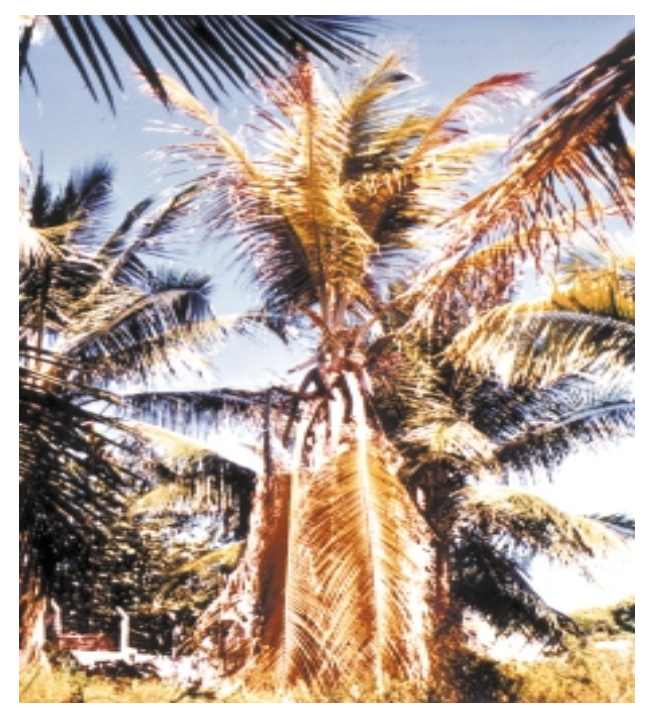

Figura 2. Sintomas interno (a) e externo (b) da doença do anel-vermelho.

\section{Aplicação do Feromônio no Campo}

Alguns testes de campo demonstraram que o isômero $(R)$ Rincoforol não possuí atividade inibitória sobre o isômero ativo $(S)$, permitindo assim a utilização no campo do racemato sintético, $( \pm)$ Rincoforol, cuja síntese é mais simples e de alto rendimento ${ }^{16}$.

$\mathrm{O}( \pm)$-Rincoforol em testes de campo ${ }^{15,17-20}$ tem demonstrado uma maior eficiência na captura dos insetos, quando associado com pedaços de cana-de-açúcar ${ }^{17}$, estipe do coqueiro ${ }^{20}$ ou frutos de abaca$\mathrm{xi}^{19}$. Como exemplo deste efeito sinérgico, Rochat e colaboradores ${ }^{17}$ obtiveram uma captura média de 3 insetos por armadilha quando utilizaram somente pedaços de cana-de-açúcar, ao passo que quando combinaram a cana-de-açúcar com o ( \pm )-Rincoforol (2,5 mg), foram coletados de 20 a 30 insetos por armadilhas. A utilização do feromônio isoladamente em campo, no entanto, tem demonstrado pouca atratividade na captura dos insetos ${ }^{15}$.

Desde 1995, o Laboratório de Ecologia Química do Departamento de Química da UFAL vem fornecendo o feromônio aos agricultores, obtido de acordo com a metodologia de trabalho aqui registrada, juntamente com instruções sobre como utilizá-lo. Hoje, o fornecimento para testes já atinge, além de Alagoas, os Estados do Espírito Santo, Bahia, Sergipe, Pernambuco, Paraíba, Rio Grande do

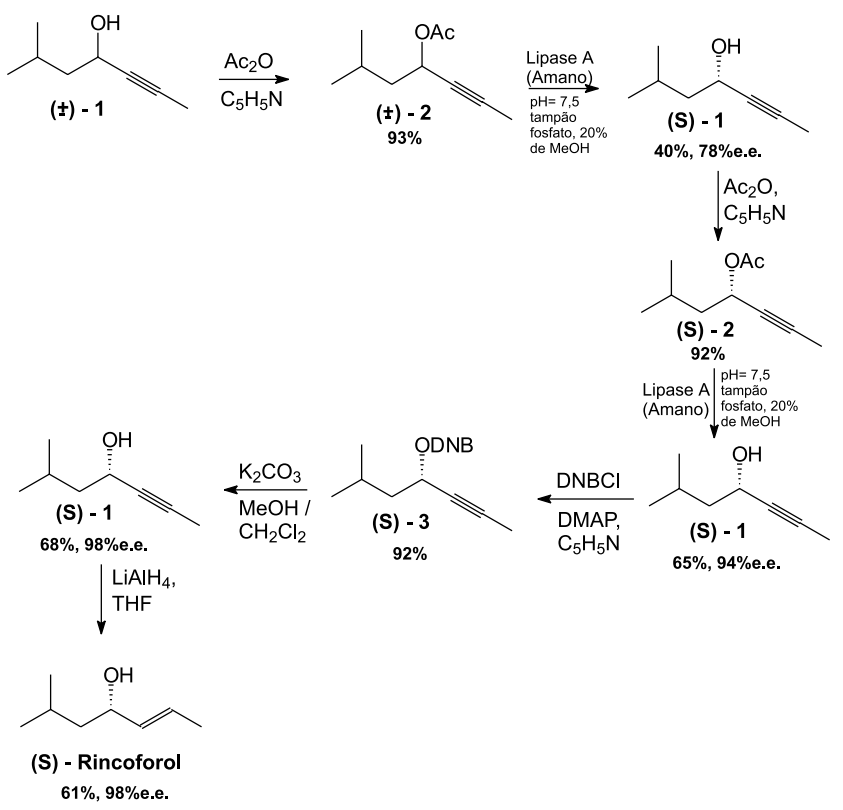

Esquema 1. Síntese assimétrica do S(-)(2E)-6-metil-2-hepten-4-ol (S)Rincoforol, feromônio de agregação do Rhynchophorus palmarum $L$. (Segundo K. Mori ${ }^{16}$ )

Norte, Piauí, Tocantins, Pará, Minas Gerais, Rio de Janeiro e São Paulo. A rota sintética que vem sendo utilizada para a obtenção do ( \pm )Rincoforol envolve uma única etapa: a adição do reagente de Grignard preparado a partir do brometo de isobutila e magnésio metálico ao crotonaldeído, como representado no Esquema 2. A demanda atual é pequena e para testes utilizamos o $( \pm)$-Rincoforol (cada cápsula contém $0,7 \mathrm{~mL} ; \mathrm{d}=0,8626 \mathrm{~g} / \mathrm{mL}$ a $28^{\circ} \mathrm{C} ; 0,6038 \mathrm{~g}$ ), com uma taxa de liberação por cápsula quando perfurada de $4,3 \mathrm{mg} / \mathrm{dia}^{21}$.

\section{PARTE EXPERIMENTAL}

Para analisar o ( \pm )-Rincoforol utiliza-se um cromatógrafo a gás HP5890, série II com detector de ionização de chama e coluna capilar (dimetilsiloxano, $12 \mathrm{~m}$ x 0,32 $\mathrm{mm}$ x 0,25 $\mu \mathrm{m}$ espessura do filme de fase estacionária). A análise é efetuada sob as seguintes condições: temperatura inicial de $35^{\circ} \mathrm{C}$ com velocidade de aquecimento de $5^{\circ} \mathrm{C} / \mathrm{min}$, e temperatura final de $150^{\circ} \mathrm{C}$. O injetor e detector do cromatógrafo são programados para operar nas temperaturas de $200^{\circ} \mathrm{C}$ e $250{ }^{\circ} \mathrm{C}$, respectivamente. $\mathrm{O}$ fluxo como gás de arraste é de $1 \mathrm{~mL} / \mathrm{min}$. $\mathrm{O}$ cromatografo a gás encontra-se acoplado a um detector seletivo de massas HP modelo 5970 controlado por um computador ChemStation 50070C.

Os reagentes utilizados para a síntese do feromônio são o 1bromo-2-metilpropano (MERCK), tetraidrofurano (VETEC - seco sob $\mathrm{LiAlH}_{4}$ e depois sódio metálico) e crotonaldeído (Aldrich).

\section{Síntese do ( \pm )-Rincoforol}

Uma solução 30\% de 1-bromo-2-metilpropano, 71 g (0,52 moles), em tetraidrofurano (THF) (120 mL) seco foi adicionada, gota-agota e sob forte agitação a $63 \mathrm{~mL}$ de THF seco contendo $12,6 \mathrm{~g}(0,52$ mol) de aparas de magnésio metálico. Após o término da adição, a reação foi mantida em repouso durante $20 \mathrm{~min}$. Em seguida, foi adicionada à mistura reacional, uma solução $30 \%$ de crotonaldeído em THF seco (33 g; 0,5 moles), gota-a-gota e mantendo-se a temperatura reacional a $30^{\circ} \mathrm{C}$, novamente após completada a adição a reação foi 
mantida em repouso por $20 \mathrm{~min}$. O meio reacional foi então resfriado até temperatura ambiente e hidrolisado com $400 \mathrm{~mL}$ de $\mathrm{HCl} 10 \%$. A fase orgânica foi separada, secada sob sulfato de sódio anidro e o solvente evaporado. A fase aquosa foi lavada 3 vezes com éter sulfúrico seco sob sulfato de sódio anidro e o solvente evaporado. As camadas orgânicas (etérea e THF) foram reunidas e purificadas. O produto foi obtido por destilação em um destilador horizontal sob vácuo com rendimento de $86 \%$, (esquema 2) p.e. $70,0-71,0^{\circ} \mathrm{C} / 35$ Torr. A análise do espectro de massas indicou o pico molecular 128 uma c intensidade de $2 \%$ em $70 \mathrm{ev} ; \mathrm{m} / \mathrm{z}(\%) \mathrm{M}^{+} 128(2), 95(2), 86(8), 85(5), 71(100), 57(12)$, 53(13) e 41(20). A análise de $\mathrm{RMN} \mathrm{H}^{1},\left(\mathrm{CDCl}_{3}, \mathrm{TMS} ; \delta ; 300 \mathrm{MHz}\right)$ : $0,91$ (d, J = 6,6 Hz, $3 \mathrm{H}, 7-\mathrm{H}), 0,92$ (d, J = 6,6 Hz, $\left.3 \mathrm{H}, \mathrm{CH}_{3} \mathrm{CH}\right), 1,30$ (ddd, J = 6,2; 7,4;13,5 Hz, $1 \mathrm{H}, \underline{\mathrm{H}}-5$ ), 1,37 (bl. 1H,OH), 1, 46 (ddd, J= 6,2; 7,4; 13,5 Hz 1H, 5-H) 1,61 - 1,77 (m, $1 \mathrm{H}, \underline{\mathrm{H}}-6), 1,70$ (dd, J = 1,4; $\left.6,4 \mathrm{~Hz}, 3 \mathrm{H}, \underline{\mathrm{CH}}_{3} \mathrm{CH}=\mathrm{CH}\right), 4,11(\mathrm{dt}, \mathrm{J}=7,1 ; 7,4 \mathrm{~Hz}, 1 \mathrm{H}, \mathrm{H}-4), 5,47$ (ddq, $\mathrm{J}=1,4 ; 7,1 ; 15,3 \mathrm{~Hz}, 1 \mathrm{H}, \mathrm{H}-3), 5,66(\mathrm{dq}, \mathrm{J}=6,4 ; 15,3 \mathrm{~Hz}, 1 \mathrm{H}$, H-2). - RNM - $\mathrm{C}^{13},\left(\mathrm{CDCl}_{3}\right.$, TMS, $\left.125 \mathrm{MHz}\right), \delta, 17,64 ; 22,50 ; 22,91$; 24,$55 ; 46,43 ; 71,35 ; 126,56 ; 134,68$, IV ( $\left.\mathrm{NaCl}, v \mathrm{~cm}^{-1}\right) .3383,2956$, 1670, 1466, 1367, 1159, 1062, 968.

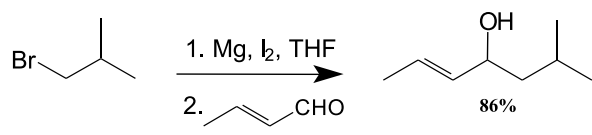

Esquema 2. Síntese do (土)-Rincoforol.

\section{Testes de Campo}

Experimentos de campo foram realizados com armadilhas contendo diferentes quantidades de cápsulas (1, 2, 4, 8 e 16 cápsulas). Estes testes possibilitaram que taxas diferenciadas do ( \pm )-Rincoforol sintético $(4,3$ a $68,8 \mathrm{mg}$ ) fossem liberadas diariamente, sem que a taxa de liberação fosse controlada.

\section{Determinação da taxa de liberação diária do Rincoforol}

Este estudo foi conduzido em casa de vegetação com o objetivo de determinar a taxa média de liberação diária do feromônio. O composto foi acondicionado em cápsulas de plástico do tipo "Eppendorf safelock $^{\circledR}$ ", contendo um orifício de $1 \mathrm{~mm}$ de diâmetro na tampa. Adicionou-se $150 \mu \mathrm{L}$ de Rincoforol em cada cápsula, as quais foram colocadas no interior das armadilhas. As observações foram realizadas diariamente, até que $50 \mu \mathrm{L}$ do feromônio evaporasse. Este experimento foi repetido dez vezes no período de 05/09/97 a 08/10/97 (33 dias). A temperatura e a umidade relativa do ar foram verificadas diariamente para efeito de comparação com as observações de campo.

\section{Obtenção da dosagem mais econômica na captura de adultos de $R$.} palmarum

De posse dos resultados sobre a taxa média de liberação diária do Rincoforol, instalou-se um experimento no campo para se observar os índices de captura de adultos de $R$. palmarum. Seis armadilhas, com diferentes taxas de liberação diária, foram instaladas em seis propriedades situadas ao longo do litoral norte do Estado de Alagoas. Neste, e nos experimentos subsequentes, as armadilhas foram enterradas no solo para facilitar o manuseio e diminuir a possibilidade de serem danificadas. A distância média entre as armadilhas foi de $5 \mathrm{Km}$, utilizando-se o delineamento estatístico do quadrado latino. As armadilhas foram inspecionadas a cada 15 dias, contandose o número de insetos machos e fêmeas capturados. Após cada inspeção, os pedaços de cana-de-açúcar eram substituídos e as armadilhas trocadas de local, para se observar o efeito do local e época na captura dos besouros. Esse experimento foi realizado no período de 26/01/98 a 27/03/98 (60 dias). Os tratamentos foram os seguintes:

1) 3 pedaços de cana-de-açúcar

2) 3 pedaços de cana-de-açúcar +1 cápsula com Rincoforol (4,3 mg/dia)

3) 3 pedaços de cana-de-açúcar +2 cápsula com Rincoforol (8,6 mg/dia)

4) 3 pedaços de cana-de-açúcar +4 cápsula com Rincoforol (17,2 mg/dia)

5) 3 pedaços de cana-de-açúcar +8 cápsula com Rincoforol (34,4 mg/dia)

6) 3 pedaços de cana-de-açúcar +16 cápsula com Rincoforol $(68,8 \mathrm{mg} / \mathrm{dia})$

\section{RESULTADOS E DISCUSSÃO}

Em casa de vegetação, a taxa média de liberação diária do Rincoforol acondicionado em cápsulas de plástico contendo um furo na tampa, foi de 5,0 $\pm 1,0 \mu \mathrm{L} / \mathrm{dia}$. Como a densidade do Rincoforol é de $0,86 \mathrm{~g} / \mathrm{mL}$, a evaporação de $5,0 \mu \mathrm{L}$ equivale a $4,3 \mathrm{mg}$. Durante os experimentos, foi mantida uma temperatura mínima diária de $18{ }^{\circ} \mathrm{C}$ e máxima de $35^{\circ} \mathrm{C}$ e umidade relativa variando entre 62 a $92 \%$.

O tratamento testemunha, somente com pedaços de cana-de-açúcar, apresentou índices de captura bem inferiores aos demais tratamentos. Os resultados demostraram que, ao utilizar armadilhas iscadas com ( \pm )-Rincoforol com taxas de liberação que, variam de 4,3 mg/dia a $68,8 \mathrm{mg} / \mathrm{dia}$, por um período de 60 dias, ou seja, experimentos de longa duração, observou-se que a taxa de $4,3 \mathrm{mg} /$ dia foi a mais econômica, uma vez que os índices de captura obtidos naquelas armadilhas, associadas ao atraente alimentar, não diferiram dos demais tratamentos (Figura 3, teste de Tukey, $\mathrm{P}<0,05)^{22}$.

Esses resultados confirmaram os dados de Oehlschlager ${ }^{18}$ nos quais foram observadas taxas de liberação de $0,3 \mathrm{mg} / \mathrm{dia}$ a $200 \mathrm{mg} / \mathrm{dia}$ por um período de 7 dias, com as armadilhas posicionadas cerca de $27 \mathrm{~m}$ uma da outra. Nosso experimento, no entanto, foi realizado por um período de 2 meses e com armadilhas separadas a uma distância de $5 \mathrm{Km}$.

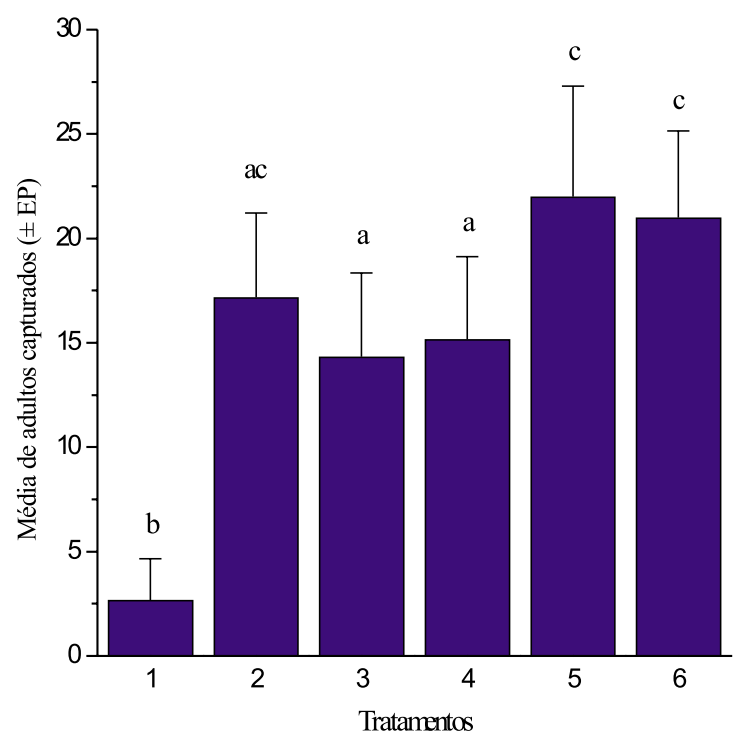

Figura 3. Número médio de adultos de R. palmarum capturados em armadilhas iscadas com cana-de-açúcar (CA) e diferentes taxas de liberação (TLR) do (士)-Rincoforol,. 1 = cana-de-açúcar, $2=C A+4,3 \mathrm{mg}$ de TLR, $3=C A+8,6 m g$ de TLR, $4=C A+17,2 m g$ de TLR, 5=CA+34,4 mg de $T L R, 6=C A+68,8 \mathrm{mg}$ de TLR. Médias seguidas da mesma letra não diferem entre si pelo teste de Tukey $(P<0,05)$. 
Através dos dados obtidos, foi possível concluir que não houve efeito significativo do local e da época na captura do $R$. palmarum como também não se observaram diferenças significantes quanto ao número de insetos machos e fêmeas capturados em cada armadilha.

\section{CONSIDERAÇÕES FINAIS}

Os testes de campo, realizados no Estado de Alagoas, demonstraram a eficiência e a qualidade do feromônio sintetizado em nossos laboratórios, além de estabelecer que apenas a utilização de uma cápsula de feromônio para uma área de 3 hectares é a opção mais eficiente e econômica para o controle do $R$. palmarum.

Tal como no Japão, onde os inseticidas vem sendo lentamente substituídos pelos feromônios, já alcançando cerca de 10-15\% do mercado, ${ }^{23} \mathrm{o}$ Brasil pode seguir este exemplo, faltando apenas mais incentivos às pesquisas na área de Ecologia Química. Esta é uma área da Química em grande expansão em todo o mundo e apresenta caráter multidisciplinar, envolvendo especialistas das áreas de biologia, química e agronomia. Esta multidisciplinaridade se deve às várias etapas envolvidas neste estudo, entre elas identificação da praga de interesse o estudo do comportamento e da biologia, o estudo dos semioquímicos relacionados ao sistema inseto-inseto e inseto-planta, o isolamento, a identificação e a determinação do(s) composto(s) ativo(s), a verificação do potencial de utilização deste(s) composto(s) no campo em armadilhas e a formulação mais eficiente para sua utilização.

\section{AGRADECIMENTOS}

Ao CNPq pelas bolsas concedidas, a Embrapa (CNPTC) e ao BNB pelo apoio financeiro. À Associação dos Plantadores de Côco de Alagoas pela divulgação e incentivo na utilização do ( \pm )Rincoforol.

\section{REFERÊNCIAS}

1. Hummel, H.E.; Miller, T.A.; Techniques in Pheromone Research; SpringVerlag; New York, 1984

2. Agellopoulos, N.; Birkett, M. A.; Hick, A. J.; Hooper, A. M.; Pickett, J. A.; Pow, E. M.; Smart, L. E.; Smiley, D. W. M.; Wadhams, L. J.; Woodcock, C. M.; Pest. Science 1999, 55, 225.
3. Harbone, J. B.; Introduction to Ecological Biochemistry; Academic Press; New York, 1993.

4. Perez, A. L.; Gries, G.; Gries, R.; Giblin-Davis, R. M.; Oehlschlager, A. C.; J. Chem. Ecol. 1994, 20, 2653.

5. Weissling, T. J.; Giblin-Davis, R. M.; Gries, G.; Gries, R.; Perez, A. L.; Pierce, Jr., H. D.; Oehlschlager, A. C.; J. Chem. Ecol. 1994, 20, 505.

6. Gries, G.; Gries, R.; Perez, A. L.; Gonzales, L. M.; Pierce, Jr., H. D.; Oehlschlager, A. C., Rhainds, M.; Zebeyou, M.; Krou, K.; J. Chem. Ecol. 1994, $20,889$.

7. Hallett, R. H.; Oehlschalagar, A. C.; Gries, G.; Angerelli, H. P. D.; Sheregi, R. K.; Gasuuma, M. S.; Borden, J.H. 1993. Field testing of aggregation pheromone of two Asian palm weevils. PORIM Internatinal Oil Palm Crongress, Kuala Lumper, Malaysia, September, 661-668.

8. Hernández, J.V.; Cerda, H.; Jaffé; Sanchez, K.; Agronomia Tropical 1992, 42,211

9. Moura, J. I. L.; Resende, M. L. V.; Sgrillo, R. B.; Nascimento, L. A.; Romano, R.; Agrotrópica 1990, 2, 165.

10. Ferreira, J. M. S.; Warwick, D. R. N.; Siqueira, L. A.; Cultura do Coqueiro no Brasil; EMBRAPA-SPI; Aracaju, 1994; p.309.

11. Griffith, R.; Plant Dis. 1987, 71, 193.

12. Moura, J. I. L.; Resende, M. L. B.; Vilela, E. F.; An. Soc. Entomol. Brasil 1995, 24, 501 .

13. Rochat, D.; Gonzalez, V. A.; Mariau, D.; Villanueva, G. A.; Zagatti, P.; J. Chem. Ecol. 1991, 17, 2127.

14. Zarbin, P. H. G.; Ferreira, J. T. B.; Leal, W. S.; Quim. Nova 1999, 22, 263

15. Oehlschlager, A. C.; Pierce, H. D., Jr.; Morgan, B.; Wimalaratne, P. D. C.; Slessor, K. N.; King, G. G. S.; Gries, G.; Gries, R.; Borden, J. H.; Jiron, L. F.; Chinchilla, C. M.; Mexzan, R. G.; Naturwissenschaften 1992, 79, 134

16. Mori, K.; Ishigami, K.; Liebigs Ann. Chem. 1992,1195.

17. Rochat, D.; Descoins, C.; Malosse, C.; Nagana, P.; Zagatti, P.; Akamou, F.; Mariau, D.; Oléagineaux 1993, 48, 225.

18. Oehlschlager, A. C.; Chinchilla, C. M.; González, L. M.; Jiron, L. F.; Mexzon, R.; Morgan, B.; J. Econ. Entomol. 1993, 86, 1381.

19. Duarte, A. G.; Lima, I. S.; Neotropical Entomol. 2001, 30, 217.

20. Weissling, T. J.; Giblin-Davis, R. M.; Scheffrahn, R. N.; Mendonza, N. M.; Fla. Entomol. 1992, 75, 212.

21. Duarte, A.G.; Dissertação de Mestrado, Departamento de Química UFAL, Maceió, 1999.

22. Bart, J.; Fligner, M. A.; Notz, W. I.; Sample and Statistical Methods for Behavioral Ecologists; Cambridge University Press; New York, 1998; p.82.

23. Hick, A. J.; Pickett, J. A.; Smiley, D. W. M.; Wadhams, L. J.; Woodcock, C.; Higher Plants as a Clean Source of Semiochemicals and Genes for Their Biotechnological Production, Phytochemical Diversity: A Source of New Industrial Product; The Royal Society Chemistry- Information Service; London, 1997. 